\title{
Plasmodium vivax lineages: geographical distribution, tandem repeat polymorphism, and phylogenetic relationship
}

Surendra K Prajapati ${ }^{1 *}$, Hema Joshi ${ }^{1}$, Sneh Shalini ${ }^{1}$, Manuel A Patarroyo ${ }^{2,3}$, Rossarin Suwanarusk ${ }^{4}$, Ashwani Kumar ${ }^{5}$, Surya K Sharma ${ }^{6}$, Alex Eapen ${ }^{7}$, Vas Dev ${ }^{8}$, Rajendra M Bhatt ${ }^{9}$, Neena Valecha ${ }^{1}$, Francois Nosten ${ }^{10,11,12}$, Moshahid A Rizvi ${ }^{13}$ and Aditya P Dash ${ }^{1,14}$

\begin{abstract}
Background: Multi-drug resistance and severe/complicated cases are the emerging phenotypes of vivax malaria, which may deteriorate current anti-malarial control measures. The emergence of these phenotypes could be associated with either of the two Plasmodium vivax lineages. The two lineages had been categorized as Old World and New World, based on geographical sub-division and genetic and phenotypical markers. This study revisited the lineage hypothesis of $P$. vivax by typing the distribution of lineages among global isolates and evaluated their genetic relatedness using a panel of new mini-satellite markers.

Methods: 18 S SSU rRNA S-type gene was amplified from 420 Plasmodium vivax field isolates collected from different geographical regions of India, Thailand and Colombia as well as four strains each of $P$. vivax originating from Nicaragua, Panama, Thailand (Pak Chang), and Vietnam (ONG). A mini-satellite marker panel was then developed to understand the population genetic parameters and tested on a sample subset of both lineages.

Results: 18 S SSU rRNA S-type gene typing revealed the distribution of both lineages (Old World and New World) in all geographical regions. However, distribution of Plasmodium vivax lineages was highly variable in every geographical region. The lack of geographical sub-division between lineages suggests that both lineages are globally distributed. Ten mini-satellites were scanned from the $P$. vivax genome sequence; these tandem repeats were located in eight of the chromosomes. Mini-satellites revealed substantial allelic diversity $(7-21, A E=14.6 \pm 2.0)$ and heterozygosity $(H e=0.697-0.924, A E=0.857 \pm 0.033)$ per locus. Mini-satellite comparison between the two lineages revealed high but similar pattern of genetic diversity, allele frequency, and high degree of allele sharing. A Neighbour-Joining phylogenetic tree derived from genetic distance data obtained from ten mini-satellites also placed both lineages together in every cluster.

Conclusions: The global lineage distribution, lack of genetic distance, similar pattern of genetic diversity, and allele sharing strongly suggested that both lineages are a single species and thus new emerging phenotypes associated with vivax malaria could not be clearly classified as belonging to a particular lineage on basis of their geographical origin.
\end{abstract}

\section{Background}

Malaria is a life-threatening parasitic disease which results in 247 million clinical episodes and nearly one million deaths annually [1]. Plasmodium falciparum is the most lethal human malaria species and causes

\footnotetext{
* Correspondence: surendramrc@gmail.com

${ }^{1}$ Molecular Biology Division, National Institute of Malaria Research (NIMR), Sector-8, Dwarka, New Delhi, India

Full list of author information is available at the end of the article
}

malignant malaria globally, while Plasmodium vivax is the most prevalent species outside Africa, causing widespread morbidity and rarely severe and fatal [2-7]. India accounts for $77 \%$ of total malaria in Southeast Asia, $P$. vivax being responsible for more than $50 \%$ of malarial cases in India annually [8].

The population genetic structure of parasite species inhabiting widely separated geographical regions defines the level of population sub-division that is the basis for

\section{Ciomed Central}


allopatric speciation. Plasmodium falciparum microsatellite studies have revealed strong bio-geographic population sub-structuring [9-11]. Likewise, $P$. vivax isolates have displayed weak population sub-structuring and shown a global population structure in several studies [12-14]. Li et al. categorized P. vivax into two distinct lineages for the first time as being Old World and New World [15] on the basis of linkage between phenotypic and genetic markers in a wide range of $P$. vivax strains. The distinction between the two lineages was (1) distinct geographic distribution, (2) differences in mosquito transmission potential, (3) fixation of the S-type polymorph from the $18 S$ small subunit ribosomal protein gene, and (4) nucleotide substitution in open reading frame 470 (orf 470) from the apicoplast genome. Plasmodium vivax isolates from the American continent were designated as New World and parasite isolates from rest of the world as Old World and they claimed that the New World lineage should be designated as a new subspecies of $P$. vivax [15].

Neutral genetic loci (tandem repeats) are considered potential genetic markers for unravelling an organism's genetic structure, population and evolutionary history. Many population studies have exploited polymorphism in two kinds of neutral loci: SNPs in putative housekeeping genes, and length polymorphism in microsatellites and mini-satellites. Genome sequence of $P$. vivax has revealed huge number of mini-satellites [16]. Genome-wide polymorphic microsatellite and mini-satellite markers have been widely used for detecting human malaria parasite population structure and genetic diversity [12,14,17-20].

The emergence of multi-drug resistance and vivax malaria-associated severe/complicated phenotypes now being reported from most vivax malaria dominated countries [2-7] could lead to the assumption that either Old World or New World parasites may have made a major contribution towards these phenotypes' emergence if both lineages (Old World and New World) are really genetically different. Furthermore, preliminary work carried out in the National Institute of Malaria Research (NIMR) in India has revealed the presence of both lineages on the Indian subcontinent [21], thus challenging Li et al's hypothesis. The established $P$. vivax lineage hypothesis has thus been questioned in this paper to uncover $P$. vivax lineages' genetic relatedness using a panel of neutral genomic markers.

\section{Methods}

\section{Parasite isolates and DNA isolation}

Four hundred and twenty Plasmodium vivax field isolates were analysed; they were collected from nine geographical populations from the Indian subcontinent $(\mathrm{N}=354)$, three Colombian regions $(\mathrm{N}=30)$, and a single Thai region $(\mathrm{N}=36)$, as well as four parasite strains each from
Nicaragua, Panama, Thailand (Pak Chang), and Vietnam (ONG). Details of individual study sites such as location, parasite and vector species prevalence and disease transmission pattern, are given in Additional file 1. Genomic DNA was extracted using QIAamp mini DNA kit (Qiagen, Germany) from microscopically diagnosed $P$. vivax-positive blood spotted on Whatman filter paper (3 mm) strips. Three punches (5 mm diameter) of dried blood spots were used for DNA isolation for each sample, as per kit manufacturer's instructions. DNA was eluted in sterile triple distilled water and stored at $-20^{\circ} \mathrm{C}$ for future use.

\section{Ethics statement}

The National Institute of Malaria Research's ethics committee approved the study protocol and all blood spots were collected with the written consent of the patients and/or and their legal guardians.

\section{Identification of mini-satellites}

Tandem Repeat Finder (TRF) software version 4.00 [22] was used for the identification of mini-satellites from $P$. vivax genome sequence. Contig-wise sequence of $P$. vivax genome was scanned for up to 30 nucleotide repeat motif. TRF scan result was filtered according to several parameters such as alignment maximum score to individual repeat motif, minimum number of Indel (insertions/deletions), unit of repeat motif, number of repeat motif (copy number), less number of mismatch in repeats and high percent identity. All the parameters were given an equal weight during mini-satellite selection and 8-15 nucleotide repeat motifs with $95-100 \%$ identity were selected.

\section{PCR amplification and tandem repeat genotyping}

A touchdown PCR method was used for the amplifying the $18 S$ SSU rRNA S-type gene from P. vivax isolates. Touchdown PCR conditions were: initial denaturation $95^{\circ} \mathrm{C} / 5 \mathrm{~min}$, and ten cycles consisting of: denaturation $95^{\circ} \mathrm{C} / 20 \mathrm{~s}(\mathrm{~s})$, annealing $65^{\circ} \mathrm{C} / 30 \mathrm{~s}$ and extension at $72^{\circ} \mathrm{C} /$ $45 \mathrm{~s}$. Annealing temperature was reduced by $1^{\circ} \mathrm{C}$ in each cycle until reaching $55^{\circ} \mathrm{C}$. At this annealing temperature $\left(55^{\circ} \mathrm{C}\right), 35$ additional cycles were run, followed by a final extension step at $72^{\circ} \mathrm{C} / 10 \mathrm{~min}$. PCR conditions for minisatellite amplification were as follows: initial denaturation $95^{\circ} \mathrm{C} / 5 \mathrm{~min}$, denaturation $95^{\circ} \mathrm{C} / 30 \mathrm{~s}$, specific annealing temperature depending on the selected marker/30s and extension at $72^{\circ} \mathrm{C} / 45 \mathrm{~s}$ for 35 cycles, and a final extension at $72^{\circ} \mathrm{C} / 10 \mathrm{~min}$. Annealing temperatures for each minisatellite and PCR primer sequences of selected genes/loci are shown in Table 1. All PCR amplification reactions were carried out in a $20.0 \mu \mathrm{L}$ final volume; $1-2 \mu \mathrm{L}(\sim 3$ $5 \mathrm{ng})$ template DNA, $10 \mathrm{pM}$ each primer, 2X Master Mix $(10.0 \mu \mathrm{L})$ (Promega or Qiagen). 
Table 1 Plasmodium vivax primers list and their annealing temperature

\begin{tabular}{|c|c|c|c|}
\hline Gene/locus & Primer name & Primer sequences $\left(5^{\prime}-3^{\prime}\right)$ & Annealing $\operatorname{Tm}\left({ }^{\circ} \mathrm{C}\right)$ \\
\hline \multirow[t]{2}{*}{ 18S SSU rRNA } & SSU-F & ATGAACGAGATCTTAACCTGC & $65 / 55$ \\
\hline & SSU-R & CATCACGATATGTA5TGATAAAGATTACC & \\
\hline \multirow[t]{2}{*}{ MiniSat-1 } & Mini1-F & ATGCTTCATTGGGTCCAC & 50 \\
\hline & Mini1-R & TCGAACAGGACAATGCTG & \\
\hline \multirow[t]{2}{*}{ MiniSat-2 } & Mini2-F & TCACCGGTGGGTCCTTCG & 50 \\
\hline & Mini2-R & GCAGCGACGAACCGTCAC & \\
\hline \multirow[t]{2}{*}{ MiniSat-5 } & Mini5-F & CAACCTGCAGAGCAATGC & 55 \\
\hline & Mini5-R & ACGTTTCTGGGCGACTTC & \\
\hline \multirow[t]{2}{*}{ MiniSat-6 } & Mini6-F & TTGTGCTGTGCTGTGCTG & 55 \\
\hline & Mini6-R & ACGGTTGGTATGGTCAGG & \\
\hline \multirow[t]{2}{*}{ MiniSat-8 } & Mini8-F & AGCCACAATCCCAACTGC & 52 \\
\hline & Mini8-R & TGGTGGTTGTGACTCTAG & \\
\hline \multirow[t]{2}{*}{ MiniSat-11 } & Mini11-F & GGCACAGTGATCATATTCG & 55 \\
\hline & Mini11-R & GCGGGTACATAACGCATG & \\
\hline \multirow[t]{2}{*}{ MiniSat-13 } & Mini13-F & GGCACATGAACTTITCGG & 52 \\
\hline & Mini13-R & TTCACCATGGTCCCTTCG & \\
\hline \multirow[t]{2}{*}{ MiniSat-14 } & Mini14-F & CTCTTCGTCGCGTCCAGG & 52 \\
\hline & Mini14-R & CAGGGTATCCACGACCAG & \\
\hline \multirow[t]{2}{*}{ MiniSat-16 } & Mini16-F & TATGTACTACCTCCACCC & 52 \\
\hline & Mini16-R & AGCGCGAATATGCATACG & \\
\hline \multirow[t]{2}{*}{ PVCDPK } & CDPK-F & CGCCTCTIITTCGAGCCC & 55 \\
\hline & CDPK-R & CTGCGCCTTCCGCGTCTT & \\
\hline
\end{tabular}

High-resolution metaphor agarose (3-4\% gel) was used for mini-satellite allele sizing. A fixed electrophoresis protocol (2 h @ 80 V) was used for all experiments for consistency in allele sizing. A 20 bp DNA ladder and Genetool software were used (SynGene Inc., UK) for allele sizing.

\section{Single clone infection typing}

As multi-clone isolates could mislead correct genotyping and lead to over-estimating genetic diversity in a multilocus genotyping study, Pvmsp-3 $\alpha$ PCR-RFLP analysis was used to identify single- and multi-clone infections [23]; only single-clone infection samples $(n=96)$ originating from five geographical regions of India (Delhi, Chennai, Nadiad, Panna and Sonapur) were used for multi-locus genotyping.

Measures of genetic diversity and phylogenetic analysis Mini-satellite genetic diversity was measured by calculating virtual heterozygosity $(\mathrm{He})$ for each locus using microsatellite analyzer (MSA) version 4.00 [24]. Virtual heterozygosity $(\mathrm{He})$ was defined as $[\mathrm{n} /(\mathrm{n}-1)]\left[1-\Sigma \mathrm{p}_{\mathrm{i}}{ }^{2}\right]$, where $\mathrm{n}$ was the number of isolates analysed and pi was the frequency of the $\mathrm{i}^{\text {th }}$ allele in the population. MEGA version $4.00[25,26]$ was used for un-rooted NeighbourJoining phylogenetic tree reconstruction method to understand the genetic relatedness of $P$. vivax $S$ type- 1 and $S$ type-2 lineages.

\section{Results}

\section{Geographic mapping of $S$ type-1 and $S$ type-2 lineages}

The 18S SSU rRNA S-type gene was amplified from 420 $P$. vivax isolates from the Indian subcontinent $(\mathrm{N}=354)$, Thailand $(\mathrm{N}=36)$ and Colombia $(\mathrm{N}=30)$ and four strains each from Nicaragua, Panama, Thailand (Pak Chang), and Vietnam (ONG). Amplified S type $18 S$ SSU rRNA DNA consisted of two PCR fragments in each sample, one having consistent size called A-type (390 bp) and a second variable sized fragment called $S$-type (either $480 \mathrm{bp}$ or $450 \mathrm{bp}$ ). $480 \mathrm{bp}$ and $450 \mathrm{bp}$ PCR fragments were designated as $S$ type- 1 and $S$ type-2, respectively (Figure 1) and served as molecular markers for identifying Old World and New World lineages of P. vivax, respectively [15]. A fair number of both $P$. vivax lineages were found on the Indian subcontinent, Thailand and Colombia; however, their distribution in geographical regions was highly variable (Figure 2 ). By contrast, two P. vivax strains from Central America (Nicaragua and Panama) were genotyped as being $S$ type-2 lineage and two strains from Southeast Asia (Thailand and Vietnam) as $S$ type-1 lineage, indicating geographical sub-division observed between Old World and New World P. vivax strains could be by chance. The $P$. vivax lineage distribution pattern for global isolates suggested a lack of parasite bio-geographic structure between New World and Old World regions; varied $P$. vivax lineage distribution throughout geographical regions was not associated with vivax or falciparum malaria or vector species prevalence (Table 2). High Old World lineage frequency was found with the Anopheles minimus malaria vector in the northeastern part of India and Thailand; however, this pattern 


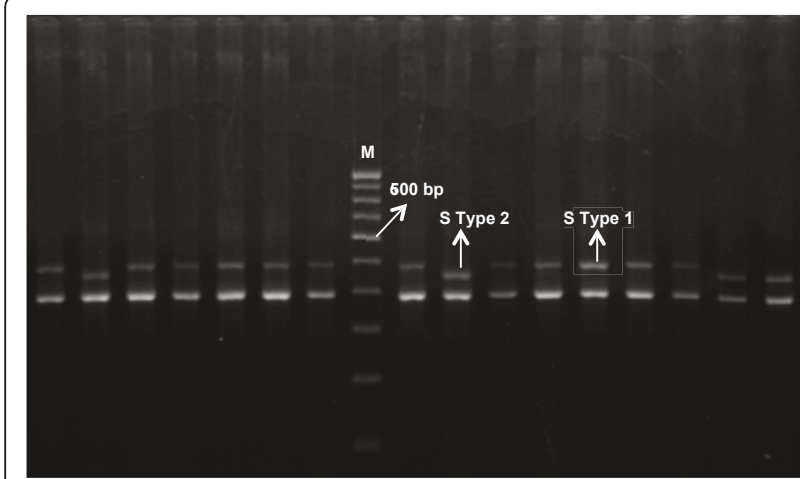

Figure $1 \mathrm{Gel}$ image of $18 \mathrm{~S}$ SSU rRNA S-type gene polymorphism distinguishing $S$ type-1 (Old World lineage) and $S$ type-2 (New World lineage).

was not consistent since one study site from Colombia revealed 100\% Old World-type prevalence with different malaria vectors (Anopheles albimanus and Anopheles darlingi).

\section{Pattern of genetic diversity between $S$ type- 1 and}

\section{$S$ type-2 lineages}

Ten mini-satellites were scanned from the $P$. vivax genome sequence; these tandem repeats were located in eight of the chromosomes (Table 3). All selected mini-satellites were highly polymorphic (Figure 3 ). A substantial number of alleles per locus $(7-21, A E=14.6 \pm 2.0)$ were observed at all mini-satellites under study except for a single locus (Minisat 8), which showed seven alleles. However, average heterozygosity was very high at all studied loci in $P$. vivax field isolates $(H e=0.697-0.924, A E=0.857 \pm 0.033)$.

Allelic diversity, frequency distribution and sharing as well as heterozygosity were compared between $P$. vivax lineages. Total allelic diversity of mini-satellites was high and similar in both lineages. A high degree of alleles in mini-satellites $(66 \%, 96 / 146)$ was shared between both lineages (Figure 4). Allele frequency per locus varied considerably; however, allele frequencies were evenly distributed between the lineages (Figure 5). Heterozygosity analysis revealed a similar mini-satellite genetic diversity pattern between both lineages (Figure 6). Thus, allelic diversity, sharing, distribution frequency and heterozygosity between both lineages revealed a similar pattern; however, a high degree of genetic diversity could only be expected from older species compared to newly evolved/established species.

\section{Genetic relationship of $S$ type-1 and $S$ type-2 lineages of $P$. vivax}

Unrooted Neighbour-Joining phylogenetic trees were constructed using 10 mini-satellite multi-locus genetic distance data. The phylogenetic tree showed five clusters having a high degree of divergence among isolates; however, no cluster was specific for any of the two lineages (Figure 7), indicating no distinction between both lineages. As per Li et al's hypothesis, it was assumed that

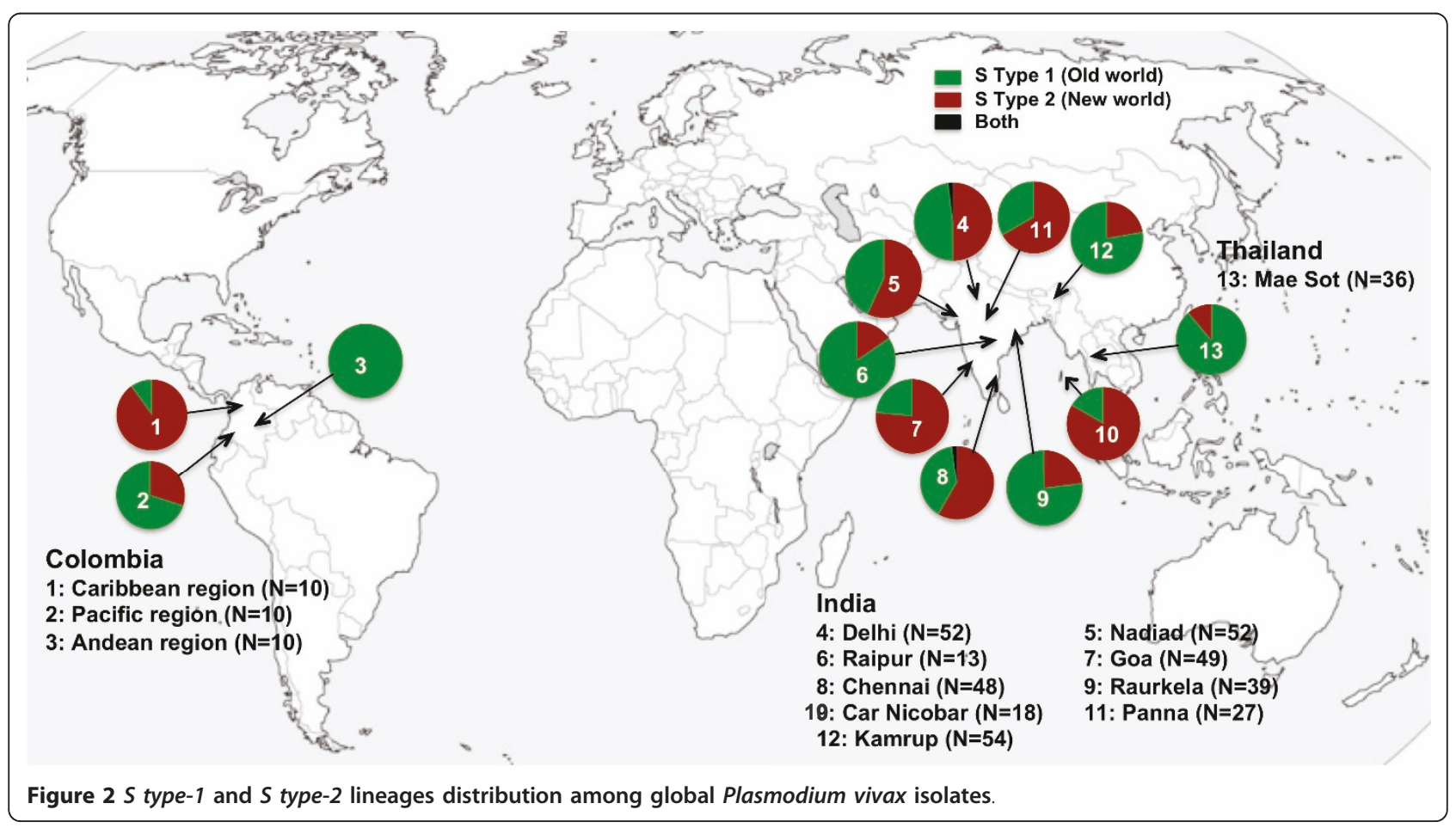


Table 2 Regional distribution of $S$ type-1 and $S$ type-2 lineages and malaria vector prevalence

\begin{tabular}{|c|c|c|c|c|c|}
\hline Regions & Major malaria parasites and vectors & Sample (n) & S type-2 (\%) & S type-1 (\%) & Both (\%) \\
\hline \multicolumn{6}{|l|}{ India } \\
\hline Delhi & Pf \& Pv, An. stephensi, An. culicifacies & 52 & 50 & 48.08 & 1.92 \\
\hline Nadiad & Pf \& Pv, An. culicifacies, An. stephensi & 52 & 55.76 & 42.3 & 1.92 \\
\hline Panna & Pf \& Pv, An. culicifacies, An. fluvitalis & 27 & 66.66 & 33.34 & - \\
\hline Raipur & Pf, An. culicifacies, An. fluvitalis & 13 & 15.38 & 84.62 & - \\
\hline Rourkela & Pf, An. fluvitalis, An. culicifacies & 39 & 23.07 & 76.93 & - \\
\hline Goa & Pf \& Pv, An. stephensi & 51 & 76.47 & 23.52 & - \\
\hline Chennai & Pv, An. stephensi & 48 & 58.33 & 39.58 & 2.08 \\
\hline Kamrup & Pf \& Pv, An. minimus, An. dirus & 54 & 22.23 & 77.77 & - \\
\hline Car Nicobar & Pf \& Pv, An. sandaicus & 18 & 83.33 & 16.67 & - \\
\hline \multicolumn{6}{|l|}{ Thailand } \\
\hline Mae Sot & Pf \& Pv, An. dirus & 36 & 11.11 & 88.89 & - \\
\hline \multicolumn{6}{|l|}{ Colombia } \\
\hline Andean region & Pf \& Pv, An. albimanus, An. darlingi & 10 & 0 & 100 & - \\
\hline Pacific region & Pf \& Pv, An. albimanus, An. neivai & 10 & 90 & 10 & - \\
\hline Caribbean region & Pf \& Pv, An. albimanus, An. nuñeztovari & 10 & 30 & 70 & - \\
\hline Total & & 420 & 46.19 & 53.09 & 0.72 \\
\hline
\end{tabular}

Both lineages' global distribution contradicted Li et al's Old and New World lineages hypothesis; however, the sympatric distribution of lineages was not sufficient to refute the two-lineage hypothesis; rather, understanding their genetic relatedness would seem to be more important. Ninety-six single-clone isolates (48 $S$ type-1 and $48 S$ type-2) were selected and characterized using a panel of mini-satellite markers to unravel genetic relatedness between $P$. vivax lineages.

$S$ type-1 and $S$ type-2 isolates would have their separate phylogenetic cluster.

\section{Discussion}

This study reports large-scale geographical mapping of the two Plasmodium vivax lineages on global isolates (Indian subcontinent, Thailand, and Colombia) and their genetic relatedness using a panel of neutral genomic markers. This study revealed that both $P$. vivax lineages have a global distribution and that their genetic structure has not been maintained between Old World and New World regions, and strongly suggests that both lineages are a population from a single species.

The global distribution of P. vivax Old World and New World lineages clearly demonstrates a lack of geographical subdivision; however, it cannot be concluded that they form a single species based only on a lack of geographical subdivision, as both lineages may have attained geographical sympatricity due to a gene flow mechanism. Their genetic relatedness was characterized by using $48 \mathrm{~S}$ type-1 and $48 S$ type-2 lineage samples to rule this out. The genetic relatedness analysis showed that both proposed genetic lineages were from the same $P$. vivax population based on the lack of genetic distance, similar genetic diversity magnitude, and no separate phylogenetic clade/cluster. As per Li et al., it was assumed that $S$ type-2 lineage (New World) is a recently evolved species and it was expected that $S$ type-2 lineage would be less diverse, and form a separate phylogenetic clade, if the hypothesis was correct. However, the panel of genomic markers consistently revealed similar patterns with all parameters used, providing no support for the hypothesis that $S$ type-2 lineage is a subspecies of $P$. vivax. This clearly suggested that $S$ type $18 S$ SSU rRNA gene polymorphism (S type-1 and $S$ type-2)

Table 3 Characteristic features of Plasmodium vivax mini and microsatellite markers

\begin{tabular}{llllll}
\hline Locus name & Chro No. & Repeat size & Repeat Unit & Copy No. & Size (bp) \\
\hline PvCDPK & 4 & 12 & ATTTGCTTCC & 25 & 375 \\
MiniSat 1 & 2 & 15 & TाTTCCCCATCTCA & 12.3 & 279 \\
MiniSat 2 & 2 & 11 & AACAAAAAAAA & 15.6 & 255 \\
MiniSat 5 & 2 & 15 & GGGGAGAGCGGCAAA & 10.1 & 254 \\
MiniSat 6 & 10 & 11 & TाTCTTCCT & 11.7 & 172 \\
MiniSat 8 & 10 & 12 & AACCAGAAATGG & 30.2 & 404 \\
MiniSat 11 & 14 & 12 & TGCTGCTCCGAC & 12.8 & 197 \\
MiniSat 13 & 14 & 9 & AGGTAAGC & 11.8 & 258 \\
MiniSat 14 & 6 & 12 & GTTGCCGCCGTG & 16 & 287 \\
MiniSat 16 & 8 & 12 & ATGTACCTACTG & 16.2 & 262 \\
\hline
\end{tabular}




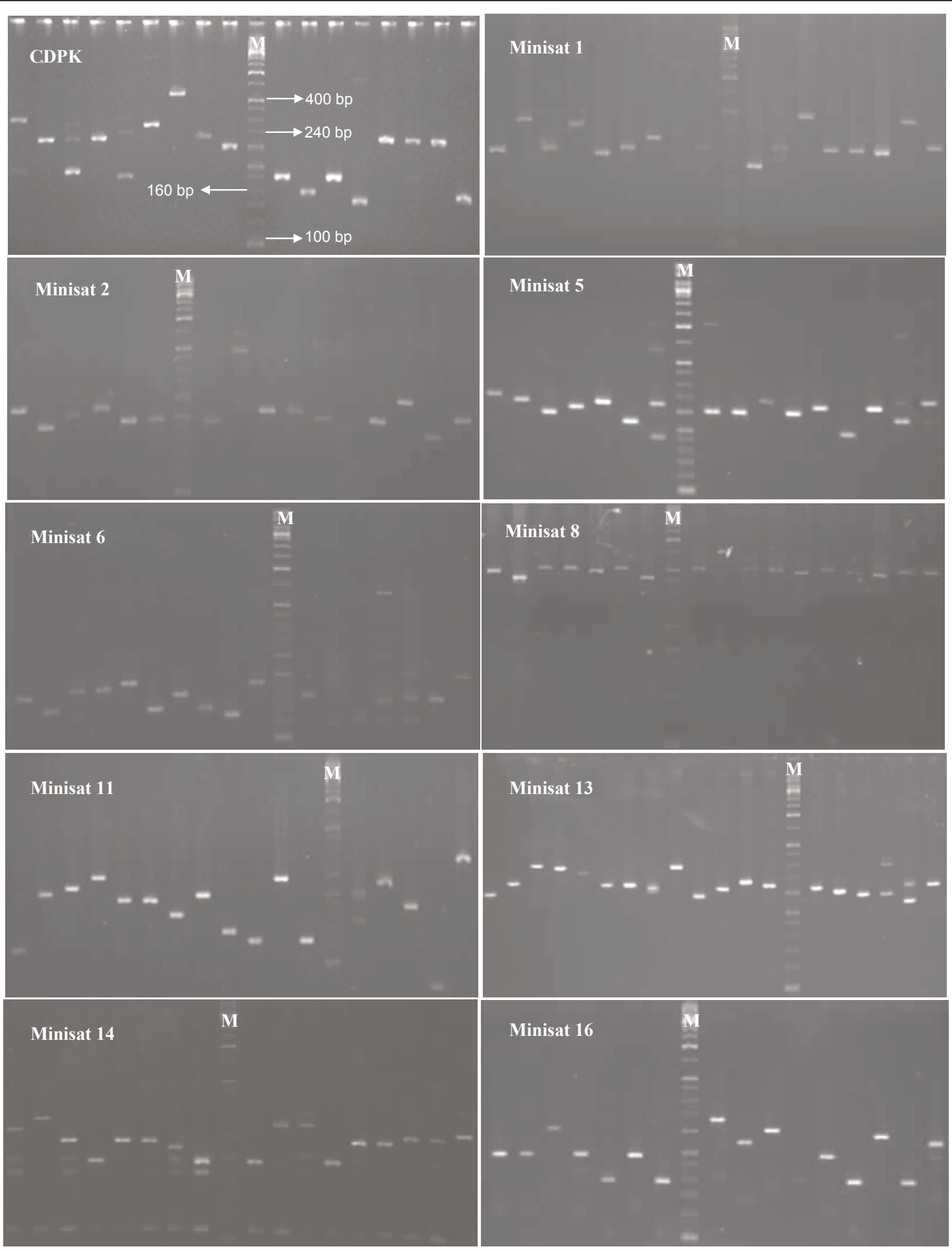

Figure $3 \mathrm{Gel}$ images of individual mini-satellite polymorphism among field isolates of Plasmodium vivax.

was not representative of two $P$. vivax lineages/sub species, rather, that both are allelic variants. The lack of significant genetic distance and population structure between $P$. vivax isolates from Old world and New World regions, in several studies, are also in strong support of the findings here reported [12-14].

Questioning of the hypothesis of Li et al. is based on contrasting findings (i.e. a lack of geographical subdivision 


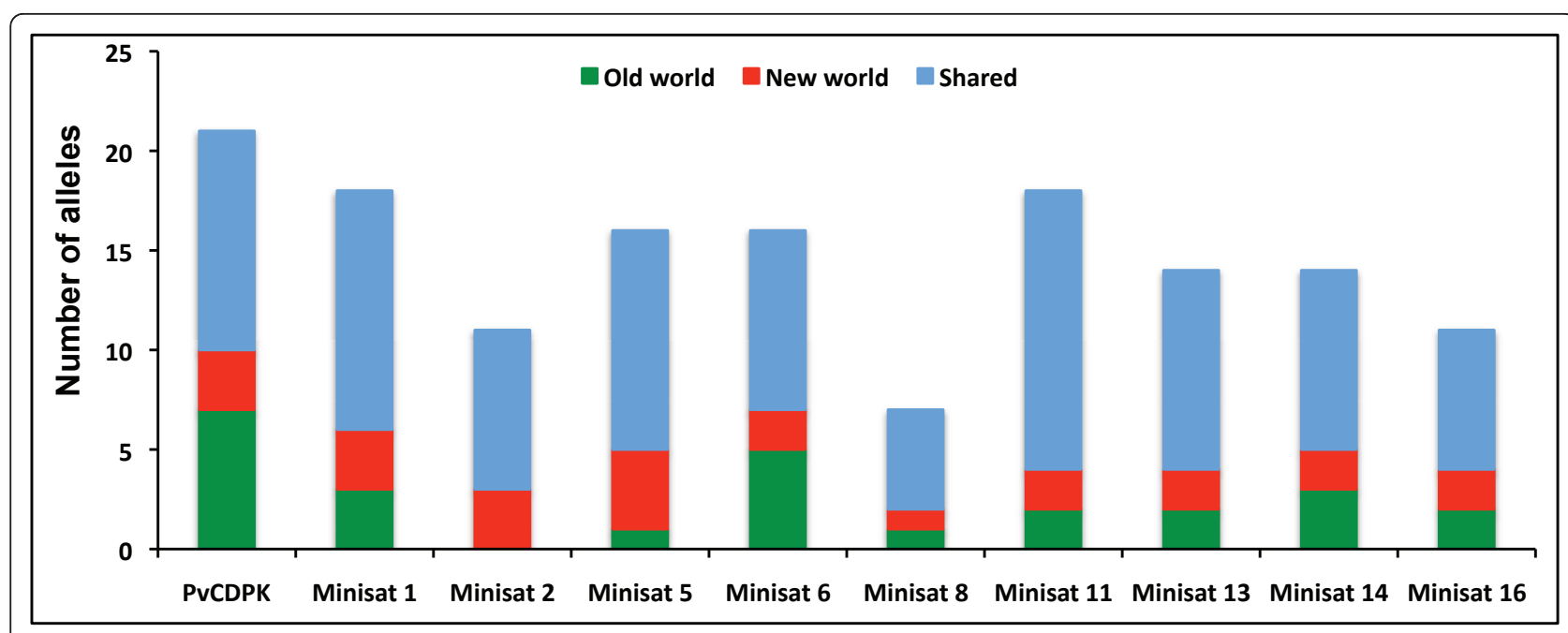

Figure 4 Allele sharing in mini-satellites between $S$ type-1 and $S$ type-2 lineages.

among global field isolates but not in parasite strains). The observations reported by Li et al. might have been due to their small sample size (17 isolates from 11 different countries) and their analysis being based only on parasite strains instead of field isolates. The regional prevalence of $S$ type- 1 and $S$ type- 2 could have been due to local vector and parasite adaptation. A recent study in Mexico by Joy et al. [19] uncovered the importance of local parasite and vector adaptation in genetic structuring of parasite populations. The effect of the mosquito's differential transmission potential, observed by Li et al., could have been the effect of local parasite-mosquito adaptation rather than speciation.

This is the first in-depth study dealing with $P$. vivax genetic diversity on the Indian subcontinent using minisatellite marker, suggesting huge neutral genetic variation in Indian field isolates. The high degree of genetic polymorphism observed in tandem repeats agrees with earlier studies, which revealed tremendous genetic polymorphism among global $P$. vivax isolates [12,14,17,18,20,27].
Although the present study has revealed a higher level of genetic diversity compared to that previously reported for $P$. vivax mini-satellites [28], this might be due to the random mating nature of Indian $P$. vivax field isolates [29]. The higher degree of genetic polymorphism displayed by Indian $P$. vivax field isolates in earlier studies [21,29-34] also supports our findings. This study successfully uncovered a substantial amount of neutral genetic variation in field isolates, implying their potential use as molecular markers in identifying population structures and relapse and recrudescence infection studies.

\section{Conclusions}

The findings have thus revealed that the Plasmodium vivax two-lineage hypothesis is unlikely; the dimorphism observed in S type 18S SSU rRNA gene represents only two alleles and both lineages are a single $P$. vivax species. The mini-satellite marker panel developed here is highly polymorphic and could be employed in therapeutic efficacy, infection relapse, and population structure studies.

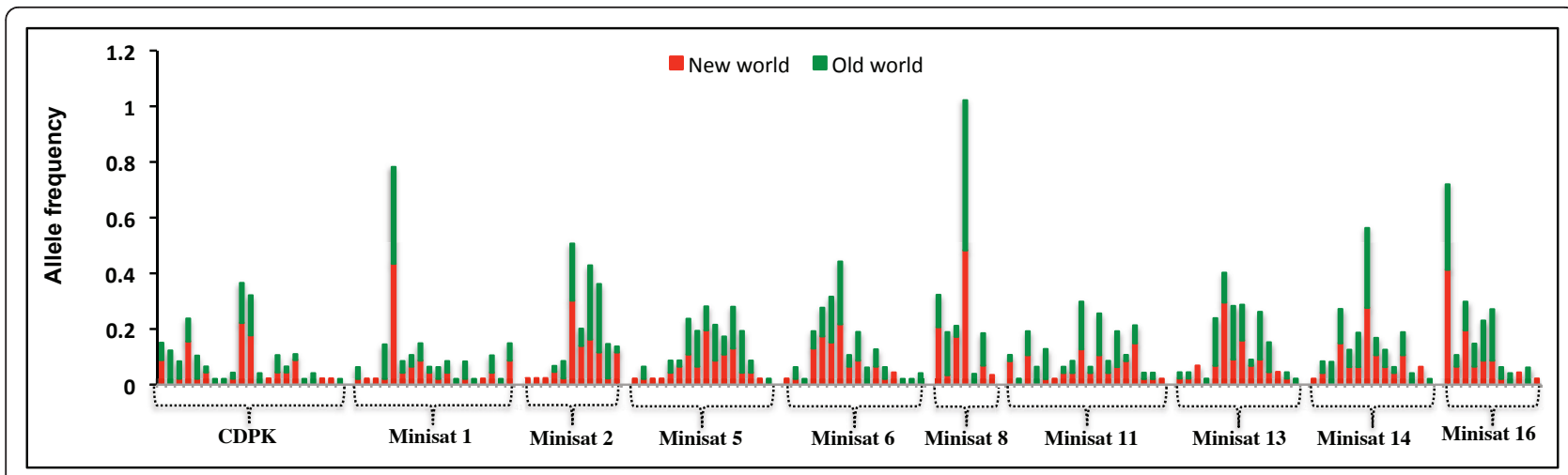

Figure 5 Allele frequency sharing at mini-satellite marker between two lineages. 


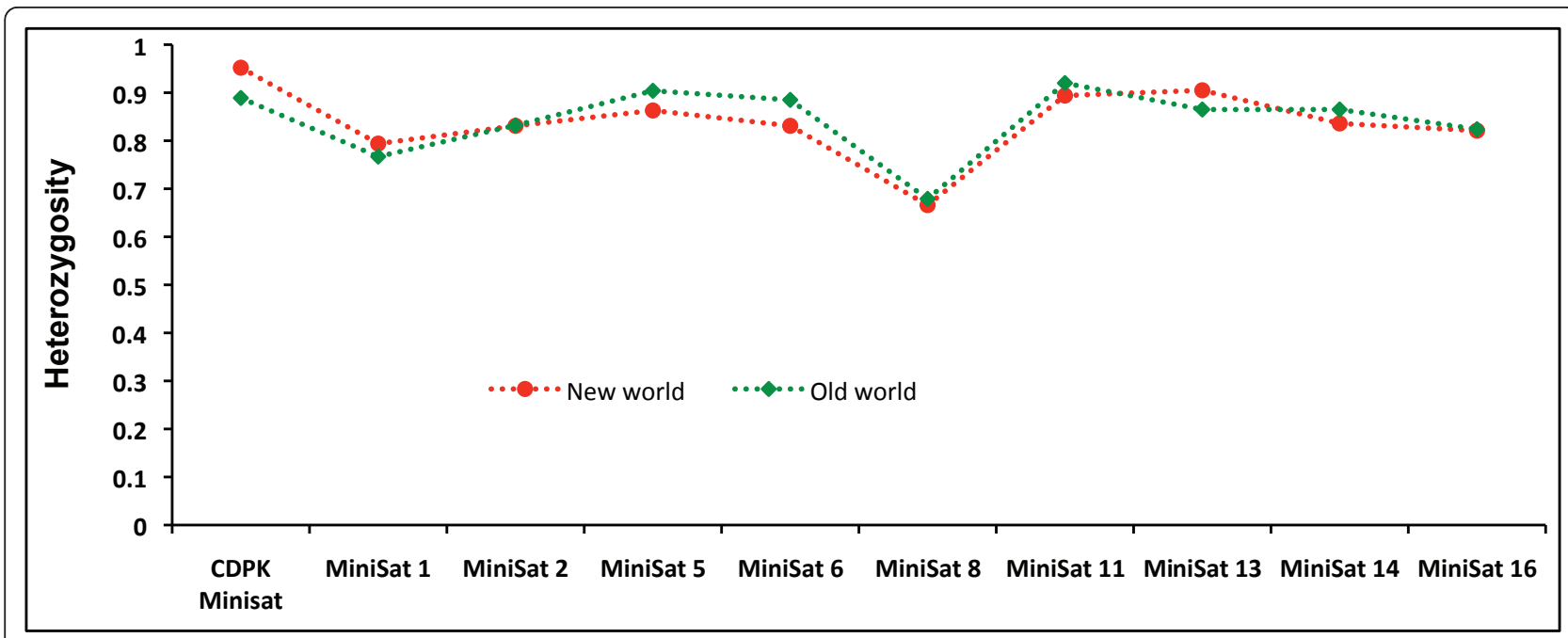

Figure 6 Degree of heterozygosity per locus in two lineages of Plasmodium vivax.

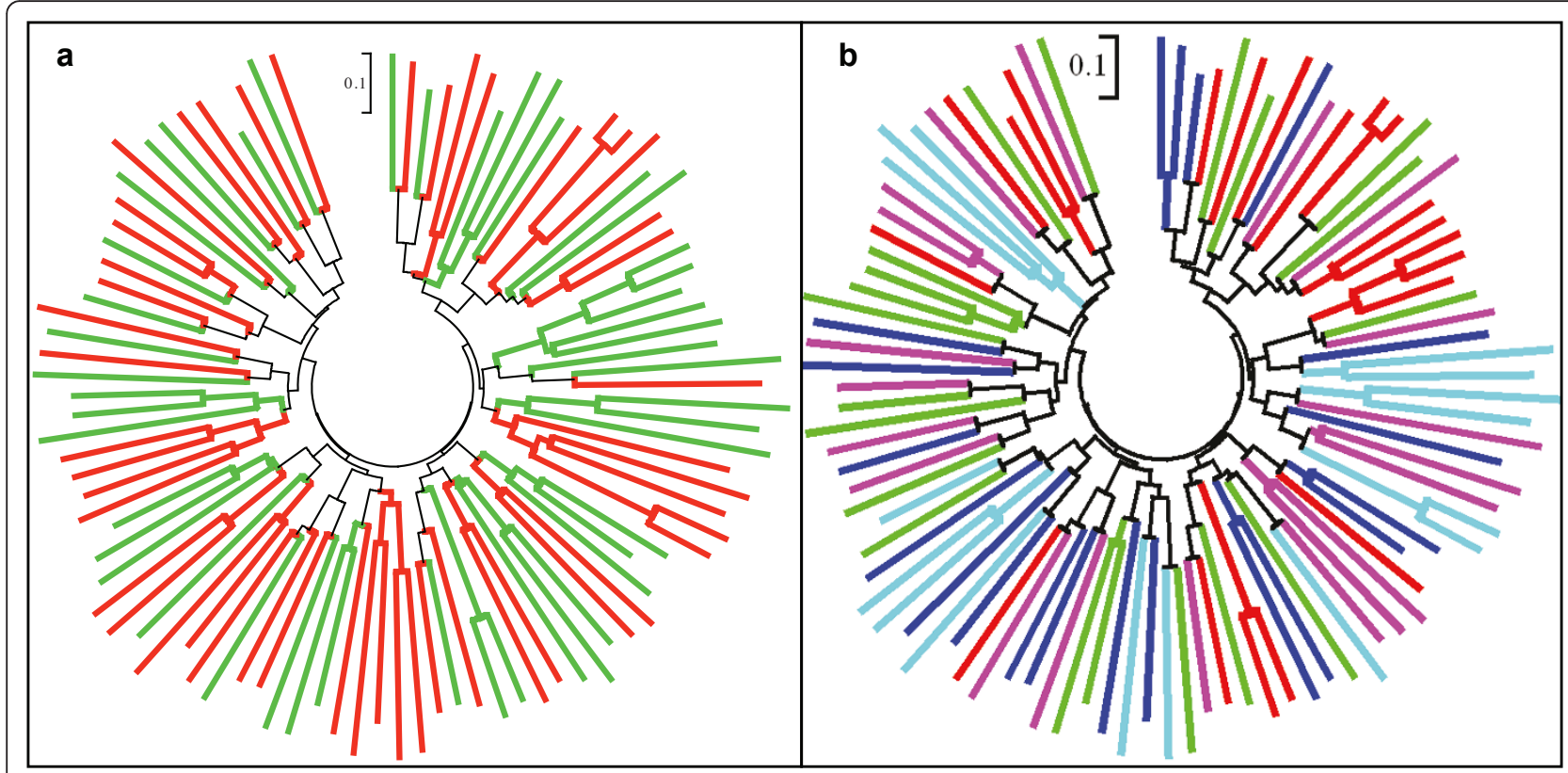

Figure $7 \mathrm{~N}$-J phylogenetic tree constructed using $S$ type-1 and $S$ type-2 lineage multi-locus microsatellite genetic distance. A: phylogenetic relationship between two lineages and, green and red lines indicate $S$ type- 1 and $S$ type-2 lineages, b: genetic structure of Plasmodium vivax isolates among five geographical regions (five colors).

\section{Additional material}

Additional file 1: Additional information

\section{Acknowledgements}

This work was supported by the Department of Biotechnology (DBT), India. SKP is an ICMR-Postdoctoral Fellow. The authors would like to thank Dr Ruchi Singh and Dr Bruce Russell for their valuable comments and suggestions regarding the manuscript, Jason Garry for correcting the English, as well as NIMR scientists, staff (Molecular Biology Division) and field units for their support and cooperation during the study and Diego GarzonOspina for providing technical assistance. The authors would like to dedicate this manuscript to the memory of Dr Hema Joshi (deceased).

\section{Author details}

'Molecular Biology Division, National Institute of Malaria Research (NIMR), Sector-8, Dwarka, New Delhi, India. ${ }^{2}$ Molecular Biology Department, Fundación Instituto de Inmunología de Colombia, Bogotá, Colombia. ${ }^{3}$ School of Medicine and Health Sciences, Universidad del Rosario, Bogotá, Colombia. ${ }^{4}$ Singapore Immunology Network (SlgN), A*STAR, Biopolis,

Singapore. ${ }^{5}$ NIMR field station, Goa, India. ${ }^{6}$ NIMR field station, Rourkela, India. 
${ }^{7}$ NIMR field station, Chennai, India. ${ }^{8}$ NIMR field station, Sonapur, India. ${ }^{9}$ NIMR field station, Raipur, India. ${ }^{10}$ Shoklo Malaria Research Unit, Mae Sot, Tak, Thailand. "'Faculty of Tropical Medicine, Mahidol University, Bangkok, Thailand. ${ }^{12}$ Centre for Tropical Medicine, Nuffield Department of Clinical Medicine, University of Oxford, CCVTM, Oxford, UK. ${ }^{13}$ Department of Biosciences, Jamia Millia Islamia University, New Delhi, India. ${ }^{14}$ World Health Organization, New Delhi, India.

\section{Authors' contributions}

SKP: Experiment design, experimental work, data analysis, manuscript writing. SS, RS, MAP: Experimental work, data analysis, manuscript writing. AK, SKS, $A E, V D, R M B, N V$, FN: Sample collection, parasite identification, and experimental design. HJ: Conceptual design and supervision of work, manuscript writing. MMAR and APD: Overall supervision of work, and manuscript writing. All authors read and approved the final manuscript.

\section{Competing interests}

The authors declare that they have no competing interests.

Received: 11 October 2011 Accepted: 19 December 2011 Published: 19 December 2011

\section{References}

1. WHO: Annual Report Geneva: World Health Organization; 2008.

2. Andrade BB, Reis-Filho A, Souza-Neto SM, Clarencio J, Camargo LM, Barral A, Barral-Netto M: Severe Plasmodium vivax malaria exhibits marked inflammatory imbalance. Malar J 2010, 9:13.

3. Kochar DK, Das A, Kochar SK, Saxena V, Sirohi P, Garg S, Kochar A Khatri MP, Gupta V: Severe Plasmodium vivax malaria: a report on serial cases from Bikaner in northwestern India. Am J Trop Med Hyg 2009, 80:194-198.

4. Kochar DK, Saxena V, Singh N, Kochar SK, Kumar SV, Das A: Plasmodium vivax malaria. Emerg Infect Dis 2005, 11:132-134.

5. Genton B, D'Acremont V, Rare L, Baea K, Reeder JC, Alpers MP, Muller I: Plasmodium vivax and mixed infections are associated with severe malaria in children: a prospective cohort study from Papua New Guinea. PLoS Med 2008, 5:e127.

6. Rogerson SJ, Carter R: Severe vivax malaria: newly recognised or rediscovered. PLOS Med 2008, 5:e136.

7. Tjitra E, Anstey NM, Sugiarto P, Warikar N, Kenangalem E, Karyana M, Lampah DA, Price RN: Multidrug-resistant Plasmodium vivax associated with severe and fatal malaria: a prospective study in Papua, Indonesia. PLoS Med 2008, 5:e128.

8. Kumar A, Valecha N, Jain T, Dash AP: Burden of malaria in India: retrospective and prospective view. Am J Trop Med Hyg 2007, 77:69-78.

9. Anderson TJ, Haubold B, Williams JT, Estrada-Franco JG, Richardson L, Mollinedo R, Bockarie M, Mokili J, Mharakurwa S, French N, Whitworth J, Velez ID, Brockman AH, Nosten F, Ferreira MU, Day KP: Microsatellite markers reveal a spectrum of population structures in the malaria parasite Plasmodium falciparum. Mol Biol Evol 2000, 17:1467-1482.

10. Anderson TJ, Roper C: The origins and spread of antimalarial drug resistance: lessons for policy makers. Acta Trop 2005, 94:269-280.

11. Anderson TJ, Su XZ, Bockarie M, Lagog M, Day KP: Twelve microsatellite markers for characterization of Plasmodium falciparum from finger-prick blood samples. Parasitology 1999, 119(Pt 2):113-125.

12. Imwong M, Nair S, Pukrittayakamee $S$, Sudimack D, Williams JT, Mayxay M, Newton PN, Kim JR, Nandy A, Osorio L, Carlton JM, White NJ, Day NP, Anderson TJ: Contrasting genetic structure in Plasmodium vivax populations from Asia and South America. Int J Parasitol 2007, 37:1013-1022.

13. Figtree M, Pasay CJ, Slade R, Cheng Q, Cloonan N, Walker J, Saul A: Plasmodium vivax synonymous substitution frequencies, evolution and population structure deduced from diversity in AMA 1 and MSP 1 genes. Mol Biochem Parasitol 2000, 108:53-66.

14. Karunaweera ND, Ferreira MU, Munasinghe A, Barnwell JW, Collins WE, King CL, Kawamoto F, Hartl DL, Wirth DF: Extensive microsatellite diversity in the human malaria parasite Plasmodium vivax. Gene 2008, 410:105-112.

15. Li J, Collins WE, Wirtz RA, Rathore D, Lal A, McCutchan TF: Geographic subdivision of the range of the malaria parasite Plasmodium vivax. Emerg Infect Dis 2001, 7:35-42.
16. Carlton JM, Adams JH, Silva JC, Bidwell SL, Lorenzi H, Caler E, Crabtree J, Angiuoli SV, Merino EF, Amedeo P, Cheng Q, Coulson RM, Crabb BS, Del Portillo HA, Essien K, Feldblyum TV, Fernandez-Becerra C, Gilson PR, Gueye AH, Guo X, Kang'a S, Kooij TW, Korsinczky M, Meyer EV, Nene V, Paulsen I, White O, Ralph SA, Ren Q, Sargeant TJ, Salzberg SL, Stoeckert CJ, Sullivan SA, Yamamoto MM, Hoffman SL, Wortman JR, Gardner MJ, Galinski MR, Barnwell JW, Fraser-Liggett CM: Comparative genomics of the neglected human malaria parasite Plasmodium vivax. Nature 2008, 455:757-763.

17. Imwong M, Snounou G, Pukrittayakamee S, Tanomsing N, Kim JR, Nandy A, Guthmann JP, Nosten F, Carlton J, Looareesuwan S, Nair S, Sudimack D, Day NP, Anderson TJ, White NJ: Relapses of Plasmodium vivax infection usually result from activation of heterologous hypnozoites. I Infect Dis 2007, 195:927-933

18. Imwong M, Sudimack D, Pukrittayakamee S, Osorio L, Carlton JM, Day NP, White $\mathrm{NJ}$, Anderson TJ: Microsatellite variation, repeat array length, and population history of Plasmodium vivax. Mol Biol Evol 2006, 23:1016-1018.

19. Joy DA, Gonzalez-Ceron L, Carlton JM, Gueye A, Fay M, McCutchan TF, Su XZ: Local adaptation and vector-mediated population structure in Plasmodium vivax malaria. Mol Biol Evol 2008, 25:1245-1252.

20. Feng X, Carlton JM, Joy DA, Mu J, Furuya T, Suh BB, Wang Y, Barnwell JW, Su XZ: Single-nucleotide polymorphisms and genome diversity in Plasmodium vivax. Proc Natl Acad Sci USA 2003, 100:8502-8507.

21. Joshi H, Prajapati SK, Verma A, Kang'a S, Carlton JM: Plasmodium vivax in India. Trends Parasitol 2008, 24:228-235.

22. Benson G: Tandem repeats finder: a program to analyze DNA sequences. Nucleic Acids Res 1999, 27:573-580.

23. Bruce MC, Galinski MR, Barnwell JW, Snounou G, Day KP: Polymorphism at the merozoite surface protein-3alpha locus of Plasmodium vivax: global and local diversity. Am J Trop Med Hyg 1999, 61:518-525.

24. Dieringer $D$, Schlötterer C: Microsatellite analyser (MSA): a platform independent analysis tool for large microsatellite data sets. Molecular Ecology Notes 2003, 3:168-169.

25. Kumar S, Nei M, Dudley J, Tamura K: MEGA: a biologist-centric software for evolutionary analysis of DNA and protein sequences. Brief Bioinform 2008, 9:299-306.

26. Kumar S, Tamura K, Nei M: MEGA: Molecular Evolutionary Genetics Analysis software for microcomputers. Comput Appl Biosci 1994, 10:189-191.

27. Gomez JC, McNamara DT, Bockarie MJ, Baird JK, Carlton JM, Zimmerman PA: Identification of a polymorphic Plasmodium vivax microsatellite marker. Am J Trop Med Hyg 2003, 69:377-379.

28. Leclerc MC, Durand P, Gauthier C, Patot S, Billotte N, Menegon M, Severini C, Ayala FJ, Renaud F: Meager genetic variability of the human malaria agent Plasmodium vivax. Proc Natl Acad Sci USA 2004, 101:14455-14460.

29. Joshi H, Subbarao SK, Adak T, Nanda N, Ghosh SK, Carter R, Sharma VP: Genetic structure of Plasmodium vivax isolates in India. Trans $R$ Soc Trop Med Hyg 1997, 91:231-235.

30. Joshi H: Markers for population genetic analysis of human plasmodia species, P. falciparum and P. vivax. J Vector Borne Dis 2003, 40:78-83.

31. Joshi H, Subbarao SK, Raghavendra K, Sharma VP: Plasmodium vivax: enzyme polymorphism in isolates of Indian origin. Trans $R$ Soc Trop Med Hyg 1989, 83:179-181.

32. Prajapati SK, Joshi H, Dua VK: Antigenic repertoire of Plasmodium vivax transmission-blocking vaccine candidates from the Indian subcontinent. Malar J 2011, 10:111.

33. Prajapati SK, Joshi $\mathrm{H}$, Valecha N: Plasmodium vivax merozoite surface protein-3 alpha: a high-resolution marker for genetic diversity studies. $J$ Vector Borne Dis 2010, 47:85-90

34. Thakur A, Alam MT, Bora H, Kaur P, Sharma YD: Plasmodium vivax: sequence polymorphism and effect of natural selection at apical membrane antigen 1 (PvAMA1) among Indian population. Gene 2008 , 419:35-42.

doi:10.1186/1475-2875-10-374

Cite this article as: Prajapati et al.: Plasmodium vivax lineages: geographical distribution, tandem repeat polymorphism, and phylogenetic relationship. Malaria Journal 2011 10:374. 